\title{
Calculation of eccentrically compressed reinforced concrete columns under various creep laws
}

Viacheslav Chepurnenko*, Elizaveta Rusakova, Irina Bugayan, Dmitriy Vysokovskiy, and Ludmila Babakhina

Don State Technical University, 344002, Rostov-on-Don, Russia

\begin{abstract}
The article proposes resolving equations for eccentrically compressed reinforced concrete short columns obtained on the viscoelastic model basis. Comparison of the results obtained according to the theory of heredity, theory of hardening, aging, flow, kinetic theory, as well as the nonlinear theory of concrete creep by Yu.A. Gurieva is presented.
\end{abstract}

\section{Introduction}

Consider a short reinforced concrete column compressed by force $F$ applied eccentrically (figure 1).

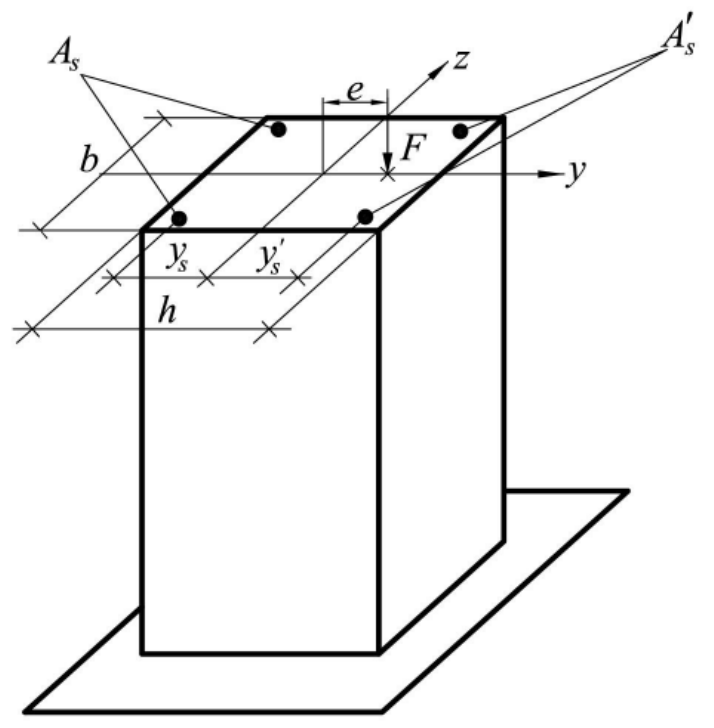

Fig. 1. Column calculation scheme

\footnotetext{
*Corresponding author: 8919888092@mail.ru
} 
The equilibrium equations for such column will be written in the form:

$$
\begin{gathered}
F=-\int_{A} \sigma_{b} d A-\sigma_{S} A_{S}-\sigma_{S}^{\prime} A_{S}^{\prime}, \\
M=F e=\int_{A} \sigma_{b} y d A-\sigma_{S} A_{S} y_{S}+\sigma_{S}^{\prime} A_{S}^{\prime} y_{S}^{\prime},
\end{gathered}
$$

where $\sigma_{b}$ are the stresses in concrete, $\sigma_{S}, A_{S}$ are the stresses and the cross-sectional area of the most compressed reinforcing bars, $\sigma_{S}^{\prime}$ and $A_{S}^{\prime}$ are the stresses and the cross-sectional area of the least compressed reinforcement, $y_{S}$ and $y_{S}^{\prime}$ is the distance from the crosssection centroid to the centers of the bars (see figure 1).

\section{Methods}

The total concrete strains in accordance with the plane sections hypothesis will be written in the form:

$$
\varepsilon_{b}=\varepsilon_{0}-y \frac{d^{2} v}{d x^{2}}
$$

where $\varepsilon_{0}$ is axial strain, $v$ is column deflection.

From the condition of compatibility of deformations of reinforcement and concrete, we obtain:

$$
\varepsilon_{S}=\varepsilon_{0}+y_{S} \frac{d^{2} v}{d x^{2}}, \varepsilon_{S}^{\prime}=\varepsilon_{0}-y_{S}^{\prime} \frac{d^{2} v}{d x^{2}} .
$$

The elastic part of concrete strain is the difference between total strain and creep strain $\varepsilon_{b}^{*}$ :

$$
\varepsilon_{b}^{e l}=\varepsilon_{b}-\varepsilon_{b}^{*}=\varepsilon_{0}-y \frac{d^{2} v}{d x^{2}}-\varepsilon_{b}^{*} .
$$

Stresses in concrete and reinforcement can be found as follows:

$$
\begin{gathered}
\sigma_{b}=E_{b} \varepsilon_{b}^{e l}=E_{b}\left(\varepsilon_{0}-y \frac{d^{2} v}{d x^{2}}-\varepsilon_{b}^{*}\right), \\
\sigma_{S}=E_{S} \varepsilon_{S}=E_{S}\left(\varepsilon_{0}+y_{S} \frac{d^{2} v}{d x^{2}}\right), \quad \sigma_{S}^{\prime}=E_{S} \varepsilon_{S}^{\prime}=E_{S}\left(\varepsilon_{0}-y_{S}^{\prime} \frac{d^{2} v}{d x^{2}}\right),
\end{gathered}
$$

where $E_{b}$ and $E_{S}$, respectively, are the elasticity modulus of concrete and reinforcement. After substituting (6) into (2) for the case of symmetric reinforcement ( $A_{S}=A_{S}^{\prime}$ and $\left.y_{S}=y_{S}^{\prime}\right)$, we get:

$$
M=-E I_{r e d} \frac{d^{2} v}{d x^{2}}-E_{b} \int_{A} \varepsilon_{b}^{*} y d A,
$$

where $E I_{\text {red }}=E_{b} I_{b}+E_{S} I_{S}$ is the stiffness of the reduced section in bending, $I_{b}=\frac{b h^{3}}{12}$, $I_{S}=A_{S} y_{S}^{2}+A_{S}^{\prime}\left(y_{S}^{\prime}\right)^{2}$.

We take from (7) the second derivative of the deflection and substituting it into (6), we obtain the formula for stresses in concrete taking into account creep: 


$$
\sigma_{b}=E_{b}\left(\varepsilon_{0}-\frac{F e}{E I_{\text {red }}} y+\frac{b y}{E I_{\text {red }}} \int_{-\frac{h}{2}}^{\frac{h}{2}} \varepsilon_{b}^{*} y d y\right) .
$$

We find the value of axial strain by substituting (6) into (1):

$$
\varepsilon_{0}=-\frac{F}{E A_{\text {red }}}+\frac{E_{b} b}{E A_{\text {red }}} \int_{-\frac{h}{2}}^{\frac{h}{2}} \varepsilon_{b}^{*} d y .
$$

To solve the creep problem, it is necessary to add an equation to expressions (8) and (9) that establishes a relationship between creep deformations and stresses. Within the framework of this article, the following creep theories are considered:

1. The theory of heredity. In accordance with the linear theory of heredity, the relationship between stresses and strains has the form [4]:

$$
\varepsilon(t)=\frac{\sigma(t)}{E(t)}-\int_{0}^{t} \sigma(\tau) \frac{\partial}{\partial \tau}\left[\frac{1}{E(\tau)}+C(t-\tau)\right] d \tau .
$$

We will restrict ourselves to considering ageless concrete, i.e. such concrete, in which the modulus of elasticity does not change over time. Then, for the creep deformation, one can write:

$$
\varepsilon^{*}=-\int_{0}^{t} \sigma(\tau) \frac{\partial}{\partial \tau}[C(t-\tau)] d \tau
$$

2. The theory of hardening. According to this theory, the rate of growth of creep strains depends on the magnitude of the creep strain at a given time, as well as the stress [5]:

$$
\frac{\partial \varepsilon^{*}}{\partial t}=\gamma\left(C_{\infty} \sigma(t)-\varepsilon^{*}(t)\right)
$$

where $\gamma$ is the coefficient, established experimentally and $C_{\infty}$ is the limiting measure of creep. If the creep measure in expression (11) has the form $C(t-\tau)=C_{\infty}\left(1-e^{-\gamma(t-\tau)}\right)$, then equation (12) is the differential form of expression (11), and the results obtained by the two theories must coincide.

3. The theory of aging. This theory explicitly establishes the relationship between creep strain, stress and time [3]:

$$
\varepsilon^{*}(t)=\sigma(t) C_{\infty}\left(1-e^{-\gamma t}\right)
$$

4. The theory of flow. The flow theory equation has the form [3]:

$$
\frac{\partial \varepsilon^{*}}{\partial t}=\sigma(t) C_{\infty} \gamma e^{-\gamma t}
$$

5. Kinetic theory. According to one of the variants of the kinetic theory [5], the relationship between the growth rate of creep strains and stresses is as follows:

$$
\frac{\partial \varepsilon^{*}}{\partial t}=\gamma \sigma(t) C_{\infty}\left(1-\frac{1}{\sigma^{2}(t) C_{\infty}} \int_{0}^{\varepsilon^{*}} \sigma\left(\varepsilon^{*}\right) d \varepsilon^{*}\right) .
$$

This article will also consider a variant of the nonlinear theory of creep of ageless concrete, proposed by Yu.A. Gurieva [1,2]. According to [1], concrete creep strain is represented as the sum of two components: $\varepsilon^{*}=\alpha_{t}+\beta_{t}$, where $\alpha_{t}=-\int_{0}^{t} \sigma(\tau) \frac{\partial}{\partial \tau}[C(t-\tau)] d \tau$ is a linear component, defined in the same way as in the theory of heredity. 
The increment in the nonlinear component of creep is assumed to be proportional to the increment in concrete damage:

$$
\frac{\partial \beta_{t}}{\partial t}=\frac{k_{2}}{R} \frac{\partial \Pi_{t}}{\partial t}
$$

where $k_{2}$ is the dimensionless coefficient.

Moreover, if $\frac{\partial \Pi_{t}}{\partial t}<0$, then $\frac{\partial \beta_{t}}{\partial t}$ is assumed equal to 0 .

The increment in material damage is considered proportional to the work of creep strains:

$$
\frac{\partial \Pi_{t}}{\partial t}=k_{1} \sigma(t)\left(\frac{\partial \alpha_{t}}{\partial t}+\frac{\partial \beta_{t}}{\partial t}\right)
$$

Eliminating the damage value from (16) and (17), we obtain the following expression for the nonlinear creep component:

$$
\frac{\partial \beta_{t}}{\partial t}=k_{1} k_{2} \frac{\sigma(t) / R}{1-k_{1} k_{2} \sigma(t) / R} \frac{\partial \alpha_{t}}{\partial t}
$$

The product of the coefficients $k_{1}$ and $k_{2}$ is usually set equal to 1 .

Consider the methodology for solving the problem. The cross section is divided in height into $m$ parts. At the first stage, the stresses for the elastic problem $\left(t=0, \varepsilon^{*}=0\right)$ are determined. We divide the time interval into $n$ steps $\Delta t$ on which the creep process is considered. If the creep law is given in differential form, then from the calculated stresses we determine the rate of growth of creep strains and the value at the next time moment:

$$
\varepsilon_{t+\Delta t}^{*}=\varepsilon_{t}^{*}+\frac{\partial \varepsilon^{*}}{\partial t} \Delta t
$$

If the creep deformation is specified explicitly as a function of stresses and time ( $\left.\varepsilon^{*}=f(\sigma, t)\right)$, as, for example, in the theory of aging, then it is determined as follows:

$$
\varepsilon_{t+\Delta t}^{*}=f\left(\sigma_{t}, t+\Delta t\right) \text {. }
$$

In the case when the creep law is given in integral form: $\varepsilon^{*}=\int_{0}^{t} K(t-\tau) \sigma(\tau) d \tau$, then the integral can be calculated numerically, for example, using the trapezoid formula:

$$
\varepsilon_{t}^{*}=\sum_{i=1}^{k} \frac{\Delta \tau_{i}}{6}\left(2 \sigma_{i} K\left(t-\tau_{i}\right)+2 \sigma_{i+1} K\left(t-\tau_{i+1}\right)+\sigma_{i} K\left(t-\tau_{i+1}\right)+\sigma_{i+1} K\left(t-\tau_{i}\right)\right), \quad \tau_{k+1}=t .
$$

In this case, the time intervals $\Delta \tau_{i}$ may not be equal to each other. The integrals included in (8), (9) and (15) are also calculated numerically using the trapezoidal method.

\section{Results and Discussion}

The problem was solved for a reinforced concrete column with the following initial data: concrete elasticity modulus $E_{b}=3 \cdot 10^{4} \mathrm{MPa}$, compressive strength of concrete $R=20 \mathrm{MPa}$, ultimate creep characteristic $\varphi_{\infty}=C_{\infty} E_{b}=3, \gamma=0.05$ days $^{-1}, k_{1}=100$, $k_{1} k_{2}=1$, cross-sectional dimensions are $b=20 \mathrm{~cm}, h=40 \mathrm{~cm}$, reinforcement elasticity modulus is $E_{S}=2 \cdot 10^{5} \mathrm{MPa}$, reinforcement compression strength $R_{s c}=400 \mathrm{MPa}$, reinforcement ratio $\mu=\frac{A_{S, t o t}}{A}=0.05, F=1200 \mathrm{kN}, e=4 \mathrm{~cm}, y_{S}=y_{S}^{\prime}=15 \mathrm{~cm}$. 
The time interval $t=100$ days was considered. The section along the height was divided into 50 parts, the time interval was divided into 100 steps.

Figure 2 shows a graph of the stress dependence from time in the most compressed reinforcement. Curve 1 corresponds to the solution based on the hereditary theory, curve 3 is based on the theory of aging, 4 is the theory of flow, 5 is the kinetic theory, 6 is the theory of Yu.A. Gurieva. The results obtained on the basis of the hardening theory coincided with the solution according to the hereditary theory, which indicates the correctness of the solution.

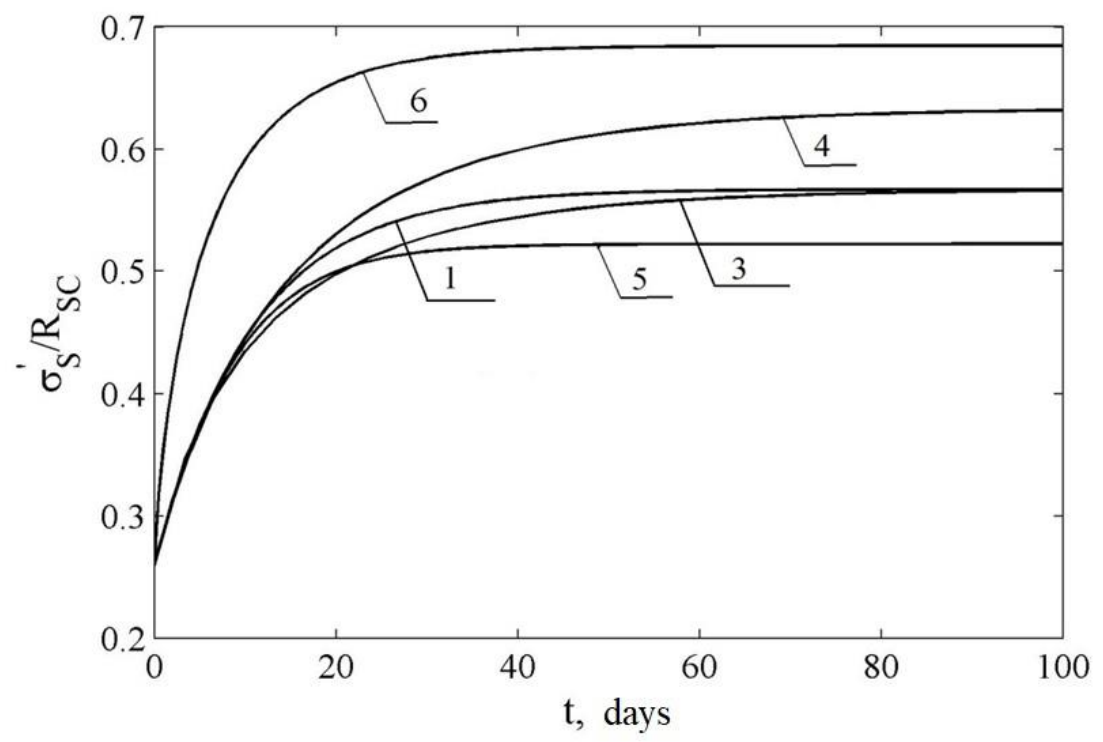

Fig. 2. Change in relative stresses in reinforcement over time

As can be seen from figure 2, the stresses in the reinforcement increase significantly over time. The largest value of stresses at the end of the creep process is obtained according to the theory of Yu.A. Gurieva, the smallest are according to the kinetic theory. Aging theory and hereditary theory give the same result on $t \rightarrow \infty$.

Figure 3 shows the stresses in concrete at the end of the creep process. The "+" sign in this graph corresponds to the compressive stresses. Dashed line is solution for $t=0$. 


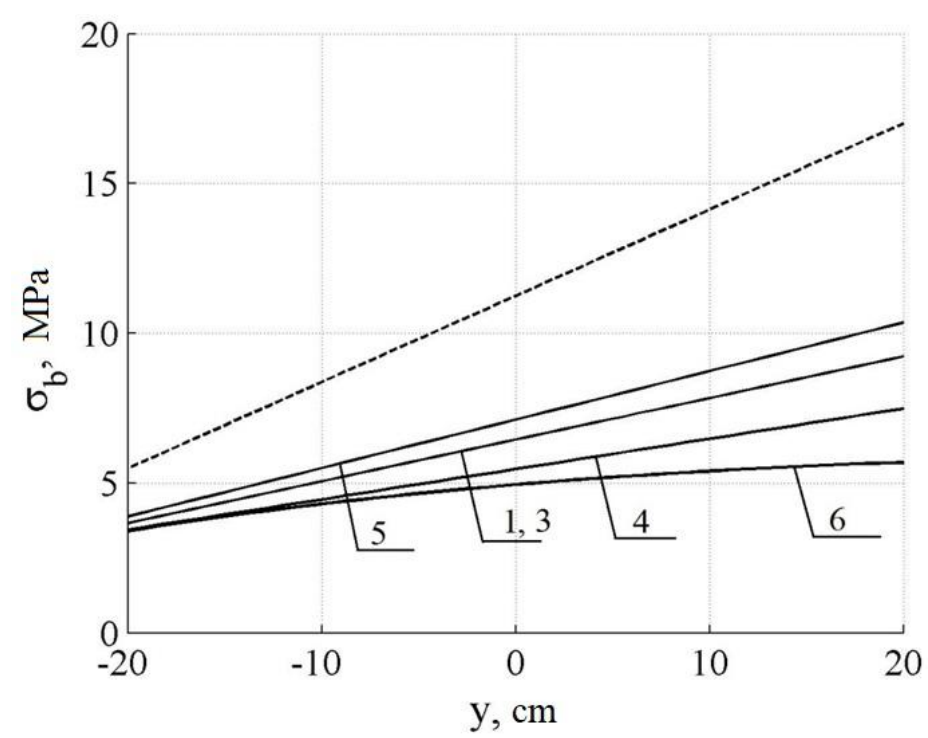

Fig. 3. Stress distribution in concrete at the beginning and at the end of the creep process

According to theories 1-5, the stresses at the end of the creep process vary linearly along the section height. The theory of Yu.A. Gurieva gives a nonlinear stress plot. Figure 3 shows that the stresses in concrete decrease over time, i.e. redistribution of stresses from concrete to reinforcement occurs. A similar effect is observed not only for bar elements, but also for shell reinforced concrete structures [6-10].

The calculation was also performed with the same initial data, but with a reinforcement ratio of 0.03 . Figure 4 shows change in the relative stresses $\frac{\sigma_{S}^{\prime}}{R_{S C}}$ in the reinforcement over time at $\mu=0.03$.

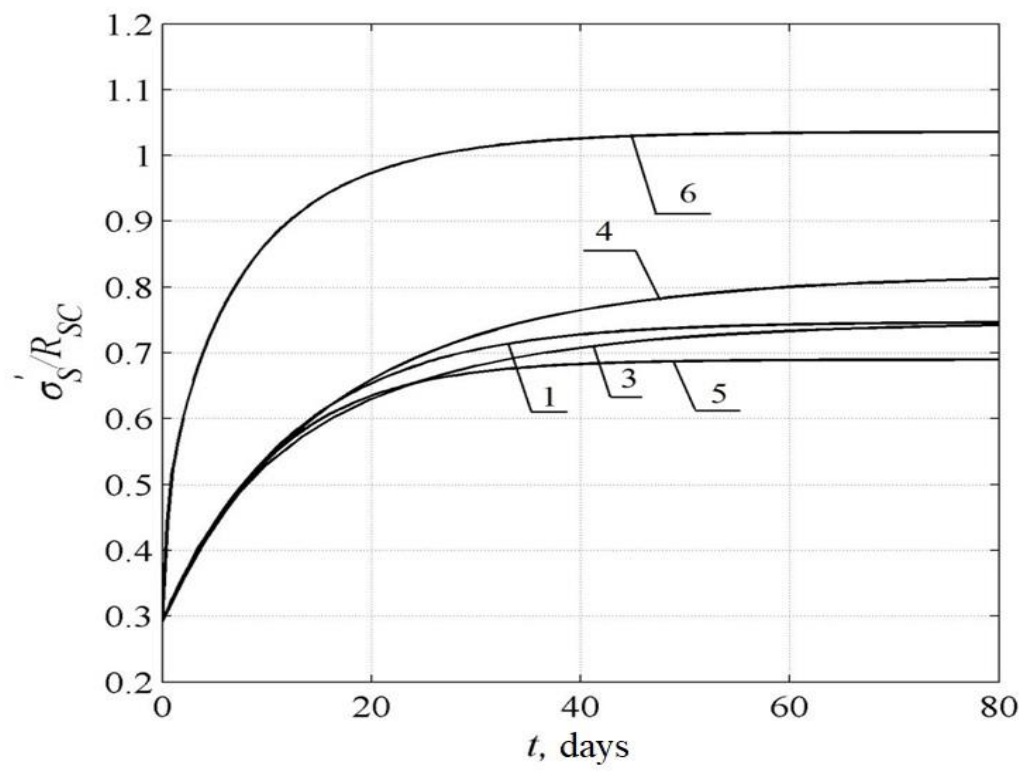

Fig. 4. Change in the relative stresses in the reinforcement over time at $\mu=0,03$ 
Here, the stresses in the reinforcement have increased, and, according to the nonlinear theory, they can reach the design resistance.

Since creep leads to an increase in material damage, the determining factor is the magnitude of the relative stresses equal to the ratio of the actual stresses $\sigma_{b}$ in concrete to the strength at a given time $r_{t}$, and not the magnitude of the true stresses in concrete. The current strength of concrete is determined as follows [1]:

$$
r_{t}=R-\Pi_{t}
$$

Figure 5 shows a graph of the change in relative stresses over time at $y=h / 2$ and $y=$ $=-h / 2$ for the reinforcement ratio $\mu=0.05$. The dashed line is the result for the linear theory of heredity, the solid line is for the nonlinear theory.

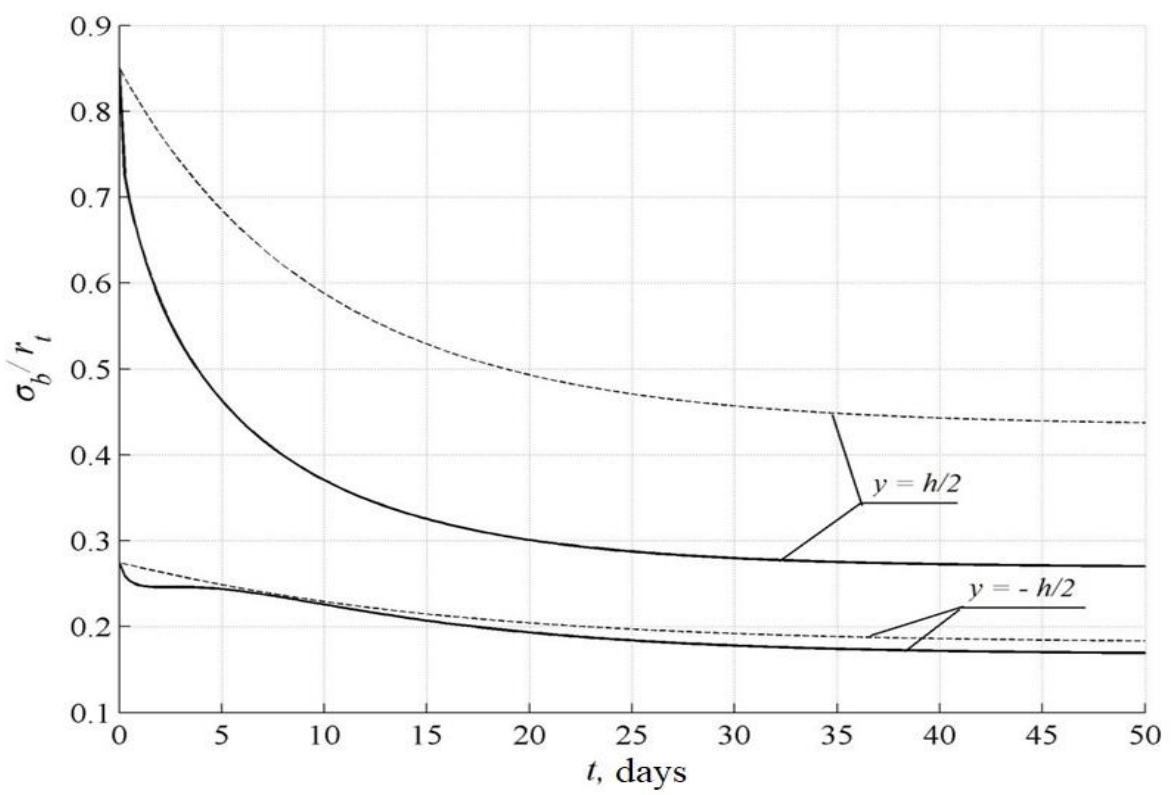

Fig. 5. Change in relative stresses in concrete over time

\section{Conclusion}

The resolving equations for eccentrically compressed reinforced concrete short columns are obtained on the basis of the model of concrete as a viscoelastic material. These equations are universal, i.e. are suitable for any relationship equations between creep strains and stresses.

A theoretical study of the stress-strain state of eccentrically compressed short reinforced concrete columns, taking into account creep, was carried out on the basis of the following theories: theory of heredity, theory of hardening, aging, flow, kinetic theory, as well as the nonlinear theory of creep of ageless concrete by Yu.A. Gurieva. Comparison of the results obtained according to the indicated theories is carried out. It is shown that in the process of creep, stresses in concrete decrease, while in reinforcement, on the contrary, they increase. The redistribution can be so significant that the stresses in the reinforcement can reach the yield point.

\section{References}


1. Yu.A. Gurieva. Simplified theory of nonlinear creep of non-aging concrete in compression: monograph (St. Petersburg: 2009)

2. Yu.A. Gurieva, Industrial and civil construction 6, 52-53 (2008)

3. K.A. Karapetyan, A.M Simonyan, Proceedings of the NAS RA: Technical sciences LIII (1), 27-34 (2000)

4. N.Kh. Harutyunyan, Some problems of the theory of creep (Moscow: Gostekhteorizdat, 1952)

5. Yu.A Shafranovsky, Concrete creep and shrinkage problems, 54-59 (1974)

6. A.G. Tamrazyan, S.G. Yesayan, Concrete creep mechanics: monograph (Moscow: MGSU, 2012)

7. B. M. Yazyev, A.S. Chepurnenko, A.V. Saibel, International Journal for Computational Civil and Structural Engineering, 13 (4) 140-148 (2017)

8. L.R. Mailyan, A.S. Chepurnenko, A. Ivanov, Procedia Engineering 165, 1853-1857 (2016)

9. A.S. Chepurnenko, L.R. Mailyan, B.M. Yazyev, A. Ivanov, MATEC Web of Conferences 106, 04010 (2017)

10. A.S. Chepurnenko, N.V. Neumerzhitskaya, M.S. Turko, Advances in Intelligent Systems and Computing 692, 808-818 (2017) 